\title{
Massive Ileal Bleeding Secondary to a Dieulafoy's lesion
}

\section{다)(우우}

\author{
Authors \\ Aymeric Becq ${ }^{1}$, Xavier Dray ${ }^{1}$, Giulia Boarini ${ }^{2}$ \\ Institutions \\ 1 Sorbonne University \& APHP Saint Antoine Hospital, \\ Department of Digestive Diseases, Paris, France \\ 2 Sorbonne Paris Cité University \& APHP Lariboisière \\ Hospital, Department of Surgery, Paris, France
}

submitted 10.8.2017

accepted after revision 9.10.2017

Bibliography

DOI https://doi.org/10.1055/s-0043-125144 |

Endoscopy International Open 2018; 06: E495-E497

(c) Georg Thieme Verlag KG Stuttgart · New York

ISSN 2364-3722

Corresponding author

Pr Xavier Dray, APHP Saint-Antoine Hospital, Department of

Digestive Diseases, 184 rue du Faubourg Saint-Antoine,

75571 Paris Cedex 12, France

\author{
Fax: +00-331-4928-2970 \\ xavier.dray@aphp.fr
}

\section{ABSTRACT}

Dieulafoy's lesions are a rare cause of gastrointestinal bleeding. This article presents the case of a young woman presenting with a massive ileal bleeding, secondary to a Dieulafoy's lesion. A computed-tomography angiography scan showed an arterial bleeding in the ileum and the diagnosis was confirmed by an emergency peroperative enteroscopy. Surgical treatment was performed. Dieulafoy's lesions account for $1 \%$ to $2 \%$ of acute gastrointestinal bleeding and lesions are mostly located in the upper gastrointestinal tract. This case stresses that Dieulafoy's lesions can be located in the lower gastrointestinal tract and that in such cases, diagnostic imaging and surgical treatment are often warranted.

\section{Introduction}

Dieulafoy's lesions are a rare cause of acute gastrointestinal bleeding and are usually located in the upper gastrointestinal tract. However, Dieulafoy's lesions can be located in the lower gastrointestinal tract. Diagnosis and management of bleeding lesions are challenging. We present a case of massive ileal bleeding secondary to a Dieulafoy's lesion and describe how this life-threatening situation was managed.

\section{Case report}

A 32-year-old woman was admitted for massive hematochezia. She had taken non-steroidal anti-inflammatory drugs (NSAIDs) for headache 4 days earlier. She had no significant past medical history. Physical examination was otherwise unremarkable. Her hemoglobin level dropped from $11.7 \mathrm{~g} / \mathrm{dL}$ to $7.6 \mathrm{~g} / \mathrm{dL}$ within 6 hours after admission. Biological workup comprising complete blood count, serum electrolytes, liver function tests and coagulation factor tests was otherwise unremarkable. The patient developed hypovolemic shock and was admitted to the intensive care unit, where she was administered intravenous fluids and noradrenaline ( $4 \mathrm{mg} /$ hour), as well as 3 packed red blood cells. An esophagogastroduodenoscopy and colonoscopy were performed and did not find the source of the bleeding. A computed tomography angiography scan showed an arterial bleeding in the ileum ( $\triangleright$ Fig. 1). An emergency peroperative enteroscopy confirmed presence of fresh blood with an adherent clot in the distal ileum, $40 \mathrm{~cm}$ upstream from the ileocecal valve ( $\triangleright$ Fig.2). An intestinal resection was performed. The specimen is shown in $\mathbf{F i g .} \mathbf{3}$ before removal of the adherent clot. Gross examination after removal of the adherent clot showed a single tiny ulceration ( $>$ Fig.4). Microscopic examination showed a large and tortuous artery running through the submucosa and protruding in the lumen through a mucosal defect, surrounded by normal mucosa ( $>$ Fig. 5 ). The patient recovered uneventfully.

\section{Discussion}

These findings are typical of exulceratio simplex, described by the French surgeon Georges Dieulafoy in 1898 [1]. Although the pathophysiology is poorly understood, it is believed that this arterial malformation is congenital rather than the result of a degenerative process [2]. Dieulafoy's lesions are twice as common in men as in women and are more frequent in the elderly. Cardiopulmonary and renal comorbidities are present in most patients, half of which are medicated with NSAIDs, aspirin 


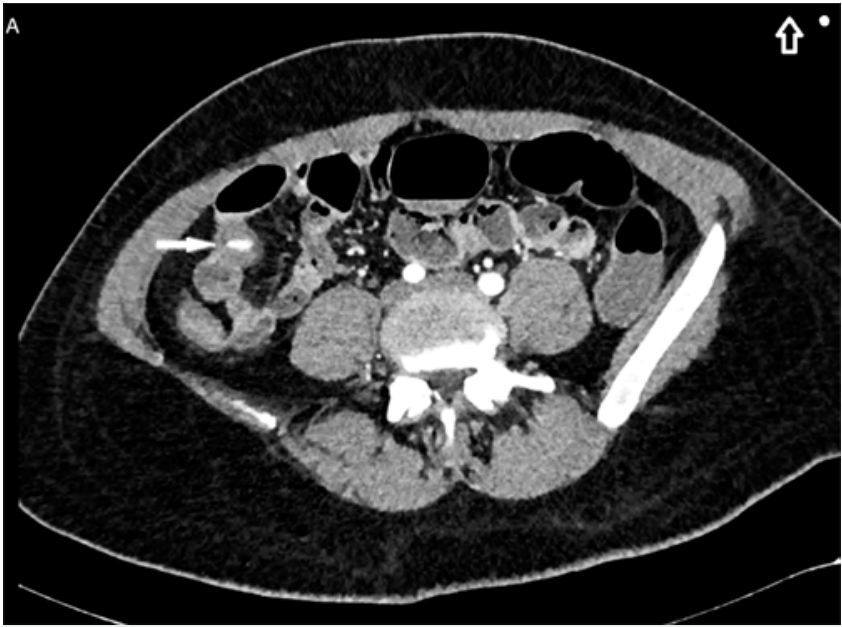

- Fig. 1 CT angiography, White arrow: Contrast extravasation compatible with arterial bleeding in the ileum.

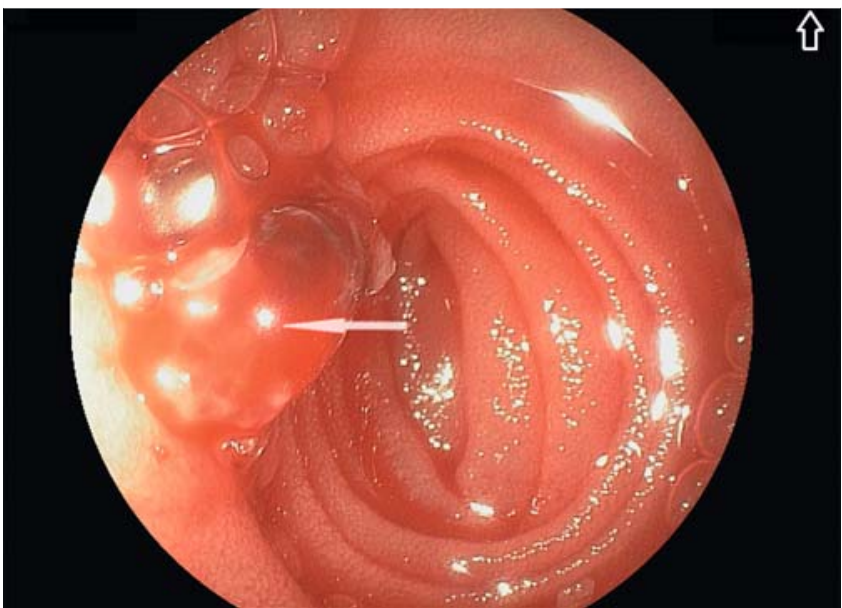

Fig. 2 Peroperative enteroscopy. Fresh blood and adherent clot (white arrow) in the distal ileum, $40 \mathrm{~cm}$ upstream from the ileocecal valve.

or anticoagulants. Mechanisms that lead to the rupture of the abnormal vessel into the digestive lumen, with subsequent abundant, spurting, gastrointestinal hemorrhage, are unknown $[2,3]$. Some authors have hypothesized that ischemic lesions and mucosal injury secondary to the use of NSAIDs may play a role in the vessel's rupture, but this remains mainly theoretical [4]. Dieulafoy's lesions account for $1 \%$ to $2 \%$ of acute gastrointestinal bleeding [2,3]. Management is challenging as the malformation is barely visible during endoscopy, aside from intermittent episodes of life-threatening spurting hemorrhage. In our patient, it is likely that the bleeding had stopped at the time of emergency surgery (spontaneously or related to the administration of noradrenaline), with constitution of a fresh, adherent clot. Lesions are located in the upper gastrointestinal tract in approximately $95 \%$ of cases: $60 \%$ in the proximal stomach (mostly within the $6 \mathrm{~cm}$ downstream the gastroesophageal junction, along the lesser curvature), $12 \%$ in the distal stomach, $15 \%$ in the duodenum and in $8 \%$ in the esophagus $[2,3]$.

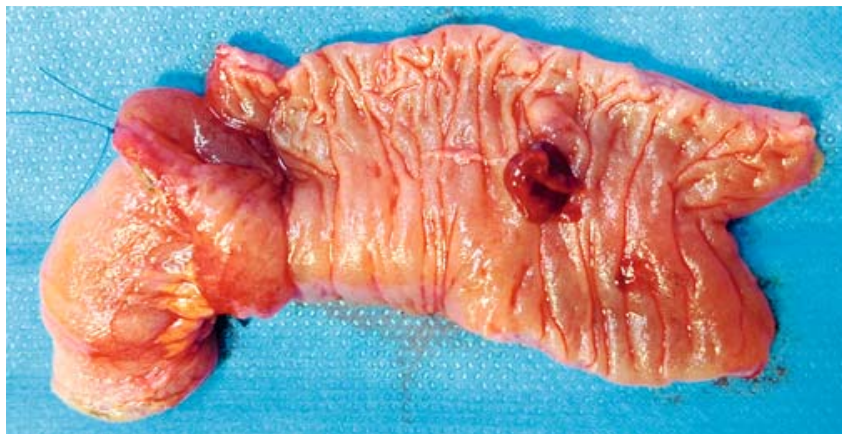

Fig. 3 Specimen after intestinal resection. Fresh clot, adherent to the wall.

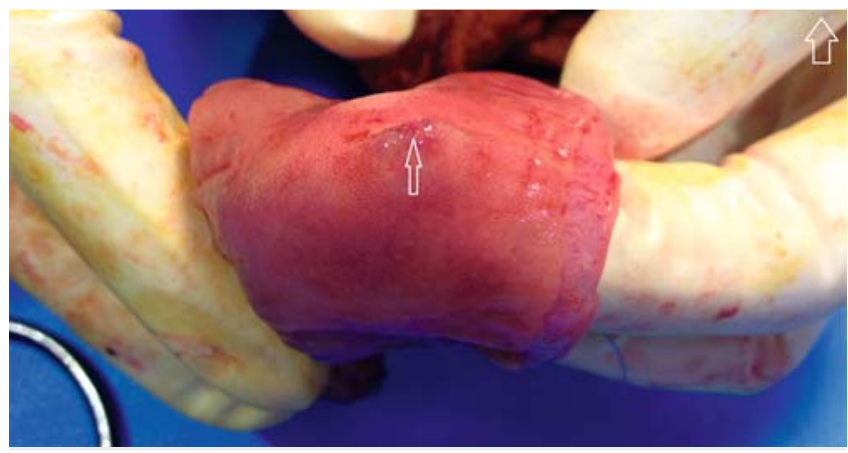

- Fig. 4 Specimen after intestinal resection, and after removal of the adherent clot. Small ulceration; exulceratio simplex.

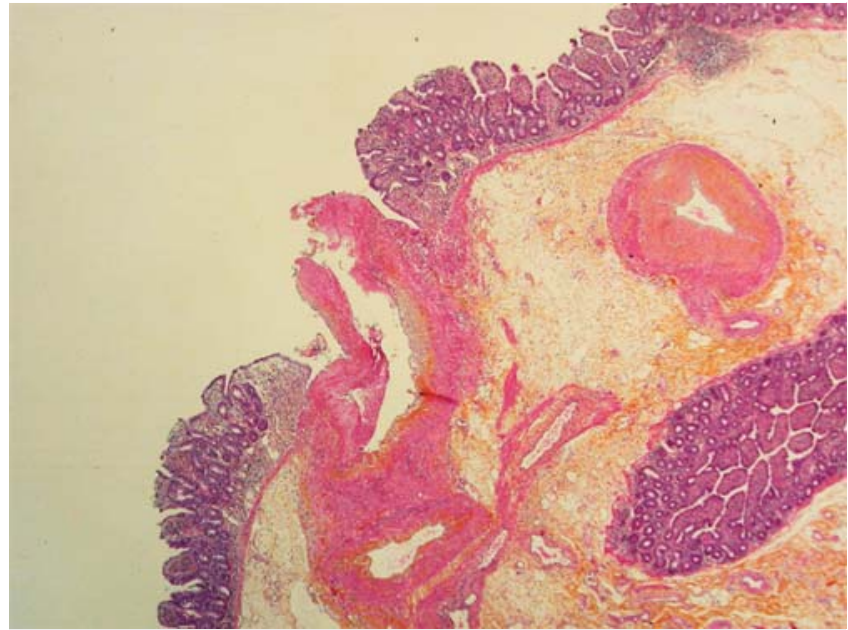

- Fig. 5 Microscopic examination. Large and tortuous artery running through the submucosa and protruding in the lumen through a mucosal defect, surrounded by normal mucosa.

Therefore, emergency upper gastrointestinal endoscopy is the key procedure for management in most cases. However, Dieulafoy's lesions are sometimes located in the lower gastrointestinal tract ( $4 \%$ in the colorectum, $1 \%$ in the jejunum and in the ileum). Endoscopic diagnosis and hemostatis (clips or electrocoagulation, possibly associated with epinephrine injection) are the cornerstone of management, whereas radiological and 
surgical approaches are considered for difficult or refractory situations.

\section{Acknowledgements}

We thank our fellow radiologists and the surgical team for helping in achieving a diagnosis and treating the patient with success.

\section{Competing interests}

Xavier Dray has acted a consultant for Boston Scientific, Fujifilm, Medtronic and Pentax.

\section{References}

[1] Dieulafoy G. Exulceratio simplex. L'intervention chirurgicale dans les hématémèses foudroyantes consécutives à l'exulceration simple de l'estomac. Bull Acad Med 1898; 49: 49-84

[2] Baxter M, Aly EH. Dieulafoy's lesion: current trends in diagnosis and management. Ann R Coll Surg Engl 2010; 92: 548 - 554

[3] Nojkov B, Cappell MS. Gastrointestinal bleeding from Dieulafoy's lesion: Clinical presentation, endoscopic findings, and endoscopic therapy. World J Gastrointest Endosc 2015; 7: 295-307

[4] Stark ME, Gostout C], Balm RK. Clinical features and endoscopic management of Dieulafoy's disease. Gastrointest Endosc 1992; 38: $545-550$ 